THE ASTROPHYSICAL JOURNAL, 559:59-69, 2001 September 20

\title{
COSMIC-RAY PROTONS ACCELERATED AT COSMOLOGICAL SHOCKS AND THEIR IMPACT ON GROUPS AND CLUSTERS OF GALAXIES
}

\author{
Francesco Miniati, ${ }^{1,2,3}$ Dongsu Ryu, ${ }^{4}$ Hyesung Kang, ${ }^{5}$ AND T. W. Jones ${ }^{2}$ \\ Received 2001 April 3; accepted 2001 May 21
}

\begin{abstract}
We investigate the production of cosmic-ray (CR) protons at cosmological shocks by performing, for the first time, numerical simulations of large-scale structure formation that include directly the acceleration, transport, and energy losses of the high-energy particles. CRs are injected at shocks according to the thermal leakage model and, thereafter, accelerated to a power-law distribution as indicated by the test particle limit of the diffusive shock acceleration theory. The evolution of the CR protons accounts for losses owing to adiabatic expansion/compression, Coulomb collisions, and inelastic $p$ - $p$ scattering. Our results suggest that CR protons produced at shocks formed in association with the process of largescale structure formation could amount to a substantial fraction of the total pressure in the intracluster medium. Their presence should be easily revealed by GLAST (Gamma-Ray Large-Area Space Telescope) through detection of $\gamma$-ray flux from the decay of $\pi^{0}$ produced in inelastic $p$ - $p$ collisions of such CR protons with nuclei of the intracluster gas. This measurement will allow a direct determination of the CR pressure contribution in the intracluster medium. We also find that the spatial distribution of CR is typically more irregular than that of the thermal gas because it is more influenced by the underlying distribution of shocks. This feature is reflected in the appearance of our $\gamma$-ray synthetic images. Finally, the average CR pressure distribution appears statistically slightly more extended than the thermal pressure.

Subject headings: acceleration of particles — gamma rays: theory — large-scale structure of universe methods: numerical - shock waves - X-rays: galaxies: clusters
\end{abstract}

\section{INTRODUCTION}

Clusters of galaxies are the largest bound objects in the universe and prove invaluable for investigations of cosmological interests. The statistics of cluster masses and their dynamical properties, including, for instance, the relative proportions of baryonic and nonbaryonic matter, are commonly used to test basic cosmological models (e.g., Bahcall 1999 and references therein). While galaxies are the most obvious constituents of clusters in visible light, most of the cluster mass is nonbaryonic, and even the baryonic matter is primarily contained within the diffuse intracluster medium (ICM) rather than in galaxies. The temperature and density distribution of the ICM gas directly reflect the dynamical state of clusters, a topic that has received much attention recently. While the ICM of clusters sometimes appears relaxed, it is often the case that high-speed flows are present, demonstrating that cluster environments can be violent (e.g., Markevitch, Sarazin, \& Vikhlinin 1999 and references therein).

The likely existence of strong "accretion" shocks several megaparsecs from cluster cores developed in the course of large-scale structure formation has been recognized for a long time (Sunyaev \& Zeldovich 1972; Bertschinger 1985; Ryu \& Kang 1997; Quilis, Ibanez, \& Saez 1998). Such shocks are responsible for the heating of the ICM up to

\footnotetext{
${ }^{1}$ Max-Planck-Institut für Astrophysik, Karl-Schwarzschild-Strasse 1, 85740, Garching, Germany; fm@MPA-Garching.MPG.DE.

${ }^{2}$ Department of Astronomy, University of Minnesota, Minneapolis, MN 55455; twj@msi.umn.edu.

${ }^{3}$ Fellow of the Network of the European Commission for the Physics of the Intergalactic Medium.

${ }^{4}$ Department of Astronomy \& Space Science, Chungnam National University, Daejeon, 305-764 Korea; ryu@canopus.chungnam.ac.kr.

${ }_{5}^{5}$ Department of Earth Science, Pusan National University, Pusan, 609-735 Korea; kang@uju.es.pusan.ac.kr.
}

temperatures on the order of $10^{7}-10^{8} \mathrm{~K}$. However, cosmic structure formation simulations have demonstrated, in addition to accretion shocks and discrete merger shocks, the existence of somewhat weaker shocks "internal" to the ICM that are very common and complex (Miniati et al. 2000). Since clusters tend to form at the intersections of cosmic filaments, they accrete matter in unsteady and nonisotropic patterns through large-scale flows propagating down filaments and producing shocks as they impact the ICM. When cluster mergers take place, the accretion shocks associated with the individual clusters add to the shocks that form in direct response to the merger. The net result of all of this is a rich web of relatively weak shocks, which often penetrate into the inner regions of the clusters (Miniati et al. 2000). Shocks resulting from discrete cluster merger events have already been identified by the observation of temperature structure in the ICM (e.g., Markevitch et al. 1999 and references therein). Such shocks have also been claimed as the acceleration sites for relativistic electrons responsible for the nonthermal emission observed from clusters in the radio, hard X-ray, and EUV bands (e.g., Takizawa \& Naito 2000; Ensslin et al. 1998; Roettiger, Burns, \& Stone 1999 and references therein).

Magnetic fields are commonly observed in the large-scale structures (e.g., Kronberg 1994). They may have been seeded at shocks in the course of structure formation and amplified up to $\mu \mathrm{G}$ level in clusters and, perhaps, also in filaments and superclusters (Kulsrud et al. 1997; Ryu, Kang, $\&$ Biermann 1998). Because shock waves in the presence of even modest magnetic fields are sites of efficient cosmic-ray (CR) acceleration (e.g., Drury 1983), structure formation might imply copious generation of high-energy particles, including both protons and electrons. In fact, according to diffusive shock acceleration theory (Drury 1983), as much as several tens of percent of the kinetic energy of the bulk flow associated with the shock can be converted into CR protons 
(Eichler 1979; Berezhko \& Ellison 1999). Given their huge size and long durability, large-scale structure shocks have also been suggested as possible sources of the very highenergy CR protons up to a few $\times 10^{19} \mathrm{eV}$ (Kang, Ryu, \& Jones1996; Kang, Rachen, \& Biermann 1997).

The overall energetics of the "cosmic" (accretion and internal) shocks is generally consistent with the production of CRs containing a significant energy fraction. Typical flow speeds in and around clusters will be $v_{f} \sim\left(2 G M_{\mathrm{cl}} / R_{\mathrm{cl}}\right)^{1 / 2} \sim$ $\left.2 \times 10^{3}\right) \mathrm{km} \mathrm{s}^{-1}$, leading to an available power for $\mathrm{CR}$ production at accretion shocks $\Phi_{E} \sim \rho_{b} v_{f}^{3} R_{\mathrm{cl}}^{2} \sim 10^{46}$ ergs $\mathrm{s}^{-1}$ using $M_{\mathrm{cl}} \sim 10^{15} M_{\odot}$ and $R_{\mathrm{cl}} \sim 2 \mathrm{Mpc}$. According to Miniati et al. (2000), where the statistics of cosmic shocks in both standard cold dark matter (SCDM) and $\Lambda$ CDM cosmologies are explored, accretion shocks appear to be less important as potential sources of CRs than internal shocks, despite the typically greater strength of the accretion shocks. The reason is that internal shocks repeatedly process the ICM material, whereas the accretion shocks do it only once with low-density background material (see Miniati et al. 2000 for $\Phi_{E}$ in clusters estimated from simulations).

Observations of radio emission from CR electrons as well as radiation excess in the hard X-ray and possibly EUV bands, have recently stimulated much discussion about cluster physics (e.g., Sarazin 1999 and references therein). They have provided information regarding the energy density of CR electrons. CR protons produce $\gamma$-rays through $\pi^{0}$ decay following inelastic collisions with gas nuclei. While such $\gamma$-rays have not yet been detected from clusters (Sreekumar et al. 1996), recent estimates have shown that $\gamma$-ray fluxes from the nearest rich clusters, such as Coma, are within the range of what may be detected by the next generation of $\gamma$-ray observatories (Colafrancesco \& Blasi 1998; Blasi 1999; Dolag \& Ensslin 2000). Their detection will provide essential information about the presence and amount of energy carried by CR protons in the ICM (see § 3.3).

Relativistic protons below the "GZK" energy threshold for photopion production owing to interaction with the cosmic microwave background photons (i.e., $E \lesssim 10^{9.5}$ $\mathrm{GeV})$ do not suffer significant energy losses in cluster environments during a Hubble time (Berezinsky, Blasi, \& Ptuskin 1997). In addition, up to somewhat lower energies $\left(\sim 10^{6} \mathrm{GeV}\right)$, even conservative estimates of diffusion rates would confine $\mathrm{CRs}$ within clusters since their formation (Völk, Atoyan, \& Breitschwerdt 1996; Berezinsky et al. 1997). Therefore, CR protons, once introduced, should accumulate in clusters, with the possibility of impacting on a wide range of issues. Some topics that could be impacted include cluster formation and evolution, as well as cluster mass estimates based on the assumption of ICM hydrostatic equilibrium. The dynamics of cooling flows also would obviously be affected.

The analysis of Miniati et al. (2000) showed that the most common shocks in the ICM have typical Mach numbers less than 10 , with a peak around $M \sim 4-5$. That is significant, because such shocks are strong enough to transfer as much as $20 \%-30 \%$ of the bulk kinetic energy into CR pressure but are only mildly modified by the CR back-reaction (Jones et al. 2000). Thus, the test-particle approximation, in which such dynamical feedback is ignored, should be a reasonable, physically justified assumption to begin investigating the production of $\mathrm{CR}$ at cosmic shocks.
In this paper we investigate the acceleration of CR protons at cosmological shocks by means of numerical calculations. For the first time the CR population is directly included in the computation with particle injection, acceleration and energy losses calculated in accord with the properties of the local environment in which the particles are propagating. Here our focus is CR protons, while CR electrons will be discussed in a companion paper (Miniati et al. 2001). There are additional sources of CRs in clusters, of course, such as active galaxies (Ensslin et al. 1997; Berezinsky et al. 1997). We do not attempt to include them in our current simulations, since our goal is to understand the role of structure shocks. However, we do call attention in our discussions to some expected differences between shock CR sources and point sources, as appropriate. The results of our modeling efforts should provide some initial clues as to how these different sources can be distinguished observationally.

The paper is organized as follows: in $\S 2$ we outline the numerical methods adopted for our study; in $\S 3$ the results are presented; a discussion on the implication of the results of this paper is given in $\S 4$; the main conclusions are summarized in $\S 5$.

\section{NUMERICAL SIMULATIONS}

\subsection{Cosmological Hydrodynamic Simulations}

For the numerical calculations we employed an Eulerian total variation diminishing hydro $+\mathrm{N}$-body cosmological code (Ryu et al. 1993). Since the computation involves a new quantity never simulated before in this context, i.e., CRs, we have decided to begin the study from the simpler case of the SCDM model, leaving the currently more favored $\Lambda$ CDM model as the natural follow-up step for future work. Although it is well known that SCDM is not a viable model anymore (e.g., Ostriker 1993), we have chosen the key cosmological parameters so that properties of the simulated collapsed objects are consistent with observations, thus allowing assessments of their general characteristics. For instance, we adopted rms density fluctuations on a scale of $8 h^{-1} \mathrm{Mpc}$ to be defined by $\sigma_{8}=0.6$, which is incompatible with $C O B E$ results and the SCDM universe, yet induces the emergence of a reasonable population of collapsed objects in simulations of large-scale structure formation (Ostriker \& Cen 1996). We have also adopted the following key parameters: spectral index for the initial power spectrum of perturbations $n=1$, normalized Hubble constant $h \equiv H_{0} /\left(100 \mathrm{~km} \mathrm{~s}^{-1} \mathrm{Mpc}^{-1}\right)=0.5$, total mass density $\Omega_{M}=1$, and baryonic fraction $\Omega_{b}=0.13$. In addition, we use a standard metal composition with hydrogen and helium mass fractions $f_{\mathrm{H}}=0.76$ and $f_{\mathrm{He}}=0.24$, respectively, which gives a mean molecular weight to be used for the temperature definition $\mu=1.22$.

In order to simulate a cosmological volume large enough to contain groups/clusters with a sufficient resolution, we select a cubic comoving region of size $50 h^{-1} \mathrm{Mpc}$ and use $256^{3}$ cells for baryonic matter and $128^{3}$ dark matter (DM) particles. This corresponds to a spatial resolution of $\sim 200$ $h^{-1} \mathrm{kpc}$. A few comments about the effects owing to finite numerical resolution are appropriate here in order to define the scope of our findings. In general, a coarse grid limits the structures that can form during the evolution of the simulated systems. This implies first that density peaks are smoothed out while masses of the structures are conserved. 
As a consequence, quantities such as the X-ray and $\gamma$-ray luminosity, which depend on the square of the density, will be reduced. The effect is stronger for lower temperature groups/clusters, which have similar structures as the larger clusters but smaller physical scales. Thus, because of resolution effects, these types of emissions (X-ray, $\gamma$-ray) will be systematically underestimated and will lead to steeper intracluster temperature dependences in our numerical calculation. Previous numerical studies carried out to test the performance of the hydrodynamic part of the code employed here indicate that the X-ray thermal emission $\left(\propto n_{\text {gas }}^{2}\right)$ is underestimated by a factor of a few (Cen \& Ostriker 1999). Second, although shocks are captured cleanly within only a few computational zones, that still amounts to a fair fraction of the cluster size. This introduces an uncertainty in the location of the shocks and reduces both the shock surface extension and complexity. However, the total flux of kinetic energy through shocks should not be affected, as indirectly attested by the fact that the computed intracluster temperatures are quite accurate (Kang et al. 1994; Frenk et al. 1999). Thus, we expect our results to be physically correct, although further work is required in order to achieve high quantitative accuracy. Since this is the first attempt to investigate such a problem, the level of accuracy characterizing our simulation should be sufficient enough to explore qualitatively the physical impact that CRs may have in a cosmological environment and to provide a preliminary assessment of their observability.

\subsection{Cosmic-Ray Injection and Acceleration}

The evolution of the CR population in the simulation is computed via passive quantities by the code COSMOCR (Miniati 2001). In the following sections we provide a brief description of the physical processes included in this code, i.e., CR injection at shocks and spatial transport and energy losses.

In the calculation, CRs are injected at shocks according to the "thermal leakage" model (e.g., Ellison \& Eichler 1984; Kang \& Jones 1995). In this model the postshock gas is assumed to have mostly thermalized to a Maxwellian distribution, $f(p)_{\text {Maxwell }}$, characterized by the downstream temperature, $T_{\text {shock }}$. Thermal protons in the high-energy tail of such a Maxwellian distribution can escape back upstream of the shock if their speeds are sufficient enough to allow them to avoid being trapped by the plasma waves that moderate the shock (Malkov \& Völk 1995). Those protons are injected into the diffusive shock acceleration mechanism and can be accelerated to high energies. In the present calculation, the momentum threshold for injection, $p_{\text {in }}$, is set to a few times the peak thermal value, i.e.,

$$
p_{\text {inj }}=c_{1} 2 \sqrt{m_{p} k_{\mathrm{B}} T_{\text {shock }}},
$$

where $m_{p}$ is the proton mass, $k_{\mathrm{B}}$ is the Boltzmann's constant, $T_{\text {shock }}$ is the postshock gas temperature, and $c_{1}$ is a parameter that regulates the number of injected particles (see below). This limit was chosen to be consistent with more detailed, nonlinear $\mathrm{CR}$ acceleration simulation results described at the end of this subsection. In the test-particle limit adopted here, the diffusively accelerated CRs emerging from a shock are characterized by a power-law distribution function given by

$$
f(p)_{\text {shock }}=f\left(p_{\text {inj }}\right) \text { Maxwell }\left(\frac{p}{p_{\text {inj }}}\right)^{-q}
$$

extending from $p_{\text {inj }}$ to $p_{\max }$. Here the log slope is determined by the shock strength, i.e., $q=3 r /(r-1)$ (where $r$ is the shock compression ratio); and the normalization is given by the value of the Maxwellian gas distribution at momentum $p_{\text {inj }}$. Thus, the thermal distribution, $f(p)_{\text {Maxwell }}$, and CR distributions, $f(p)_{\text {shock }}$, join smoothly in terms of the momentum coordinate. This power-law CR distribution is assigned to each grid cell that is identified as "being shocked " within a time step in the numerical simulation. The physical upper bound to the CR momentum distribution is determined by several factors, including the time available for acceleration compared to the mean time for particles to recross the shock owing to the competition between wave scattering and advection, the extent of a shock compared to particle scattering lengths, and any competition from energy losses during acceleration. For parameters appropriate to groups/ clusters we expect the acceleration to proceed relatively quickly up to momenta at least as great as $10^{6} \mathrm{GeV} \mathrm{c}^{-1}$. CRs with even higher energy can be produced in principle (Kang et al. 1996). Conservative estimates indicate that these very high-energy CRs can diffuse out of clusters carrying away some energy. That would affect our results only if the spectra of the accelerated CRs are significantly flattened with respect to the test particle limit above our adopted momentum upper limit. However, this type of behavior typically is expected only for CR-dominated and strongly modified shocks. From the observed properties of the ICM, where most of the pressure is thermal, that type of shock most likely does not occur.

In the simulation, we assume the power law is formed within one dynamical time step up to $p_{\max }=10^{6} \mathrm{GeV} c^{-1}$, so that spatial diffusion of CRs can be neglected and the computational cost much reduced. To follow the evolution of the CR distribution in detail from injection to $p_{\max }$ would be completely impractical, since it would necessitate numerical resolution on the scale of the physical thickness of the shocks (Jones, Ryu, \& Engel 1999). Similarly, since spatial diffusion of CRs away from shocks is likely to be slow below $p_{\max }$, it is neglected there, but COSMOCR does include adiabatic energy changes, as well as the energy losses from Coulomb and inelastic $p$ - $p$ collisions with the thermal ICM. To do this, a Fokker-Planck equation is solved that has been integrated over finite momentum bins to take advantage of the near power-law form of the CR momentum distribution, $f(p)$. In effect, the momentum space is divided into eight logarithmically equidistant intervals, bounded by $p_{1}, \ldots p_{8}, p_{9}=p_{\max }$, which we refer to here as momentum bins. Within each momentum bin, $j$, we assume $f\left(\boldsymbol{x}_{i}, p\right) \propto p^{-q_{j}\left(\boldsymbol{x}_{i}\right)}$, where $q_{j}\left(\boldsymbol{x}_{i}\right)$ is determined selfconsistently from $n\left(\boldsymbol{x}_{i}, p_{j}\right)$ defined below and the required continuity of $f(p)$. At each computational spatial grid point, $\boldsymbol{x}_{i}$, and for each momentum bin, $j$, we define the number density as

$$
n\left(\boldsymbol{x}_{i}, p_{j}\right)=\frac{4 \pi}{3} \int_{p_{j}}^{p_{j+1}} f\left(\boldsymbol{x}_{i}, p\right) p^{2} d p .
$$

For a full description of the code COSMOCR we refer to Miniati (2001, but see also Jones et al. 1999).

Before concluding this section, we return for a moment to the "injection" parameter $c_{1}$, which deserves some further comments. As already pointed out, the value of $c_{1}$ determines the fraction $\eta_{\text {inj }}$ of particles in the postshock gas with density $n_{2}$ that are injected into the CRs as follows (Miniati 
2001):

$$
\eta_{\mathrm{inj}} \equiv \frac{n_{\mathrm{inj}}}{n_{2}}=8 \sqrt{\frac{2}{\pi}} c_{1}^{3} e^{-2 c_{1}^{2}} \frac{\left(p_{\mathrm{max}} / p_{\mathrm{inj}}\right)^{3-q}-1}{3-q},
$$

where, obviously, $n_{\mathrm{inj}}$ is the number of injected particles. In addition, we find the ratio of the CR pressure to ram pressure $\left(\rho_{1} u_{s}^{2}\right.$, which supplies the energy for the CRs) to be (Miniati 2001)

$$
\begin{aligned}
\frac{P_{\mathrm{cr}}}{\rho_{1} u_{s}^{2}}= & \frac{8}{3} \sqrt{\frac{2}{\pi}} c_{1}^{3} e^{-2 c_{1}^{2}}\left(\frac{m_{p} c}{p_{\mathrm{inj}}}\right)^{3-q}\left(\frac{c}{u_{s}}\right)^{2} \\
& \times \frac{\left(p_{\max } / p_{\mathrm{inj}}\right)^{4-q}-1}{4-q},
\end{aligned}
$$

where $c$ and $u_{s}$ are the light and shock speed, respectively, and where we neglected the nonrelativistic contribution of the CR pressure (this is justified since $q \simeq 4$ so that most of the CR pressure is produced by relativistic particles; see $\S 3$ ). Both observational and theoretical studies of diffusive shock acceleration suggest that canonical values of $c_{1}$ should be around 2.3-2.5, corresponding to the value of $\eta_{\mathrm{inj}}$ ranging between a few $\times 10^{-3}$ and $10^{-4}$ (Lee 1982; Quest 1988; Kang \& Jones 1995). According to the simulations by Gieseler, Jones, \& Kang (2000), where a self-consistent injection treatment based on the plasma physical model of Malkov \& Völk (1995) is adopted, the above injection parameter is, in fact, very reasonable. For the flow parameters relevant for the cosmic shocks in our simulations, we find that a value of $c_{1}=2.6$ produces an injection efficiency $\eta_{\text {inj }}$ and a postshock CR pressure consistent with the saturation value obtained from numerical studies of shock acceleration, in which the back-reaction of the particles is accounted for (Berezhko, Ksenofontov, \& Yelshin 1995). Note that, for this reason, our value of $c_{1}$ is somewhat larger than the canonical values owing to the test-particle treatment. For a shock with Mach number $M \sim 4$ and speed $u_{s} \sim 10^{3} \mathrm{~km} \mathrm{~s}^{-1}, q \sim 4.2$, and we evaluate (for $p_{\max }=10^{15} \mathrm{eV}$ )

$$
\begin{aligned}
\frac{P_{\mathrm{cr}}}{\rho_{1} u_{s}^{2}} \sim 9.5 & \times 10^{-2}\left(\frac{c_{1}}{2.6}\right)^{4.2} e^{-2\left(c_{1}^{2}-2.6^{2}\right)} \\
& \times\left(\frac{M}{4}\right)^{-1.2}\left(\frac{u_{s}}{10^{3} \mathrm{~km} \mathrm{~s}^{-1}}\right)^{0.8} .
\end{aligned}
$$

Since most of the flow kinetic energy is processed by cosmic shocks with $M \sim 4-5$, we expect from experience with detailed CR shock simulations that up to $10 \%-30 \%$ of it will be converted into CR energy with the above value of $c_{1}$. Detailed simulations also show that modifications to such shocks are small enough that the form of the CR spectrum is not substantially changed from the test-particle theory (Jones et al. 2000). So our choice of $c_{1}$ is also consistent with our assumption that the $\mathrm{CR}$ acceleration can be treated by the test-particle theory.

\subsection{Extracting Global Properties of Groups/Clusters}

After the calculation was completed, the simulated groups/clusters have been identified by the DM-based "spherical overdensity" method described in Lacey \& Cole (1994). The details of the group/cluster identification procedure can be found in Miniati et al. (2000). Global group/ cluster properties, such as core temperature, average pressure, emissivity at various wavelengths, etc., were calculated by averaging or integrating the quantities over the group/cluster volume. These global properties have then been studied by means of correlation plots in order to make predictions about the quantities under investigation (and often yet to be measured) in terms of the well-established ones (see $\S \S 3.1-3.3$ ). We point out from the outset that because of the relatively small computational box, the temperature of the simulated collapsed objects only ranges between 0.3 and $3 \mathrm{keV}$. Nevertheless, after determining the temperature dependence of the various properties of interest, we extrapolate their values beyond these temperature limits and make estimates even for rich clusters, such as Coma, which are easier to observe. So long as there are no important scales involved, these extrapolations should be reliable.

Two-dimensional projections of individual group/cluster structures have also been constructed from the data set, either as slices through the simulated volume or as synthetic images of cluster emissions. The synthetic images, computed here in the X-ray and $\gamma$-ray bands, are produced by means of a projection code (I. Tregillis 2001, in preparation) that integrates the "emissivity" along the line of sight in the optically thin plasma approximation. These images allow a more in-depth inspection of the spatial distribution of the quantities of interests but for space reasons are limited here to only a few examples (see $\S 3.4$ ).

In general, the $\gamma$-ray flux and the surface brightness for the synthetic images have been calculated by arbitrarily setting the groups/clusters to a luminosity distance of about $70 h^{-1}$ Mpc (i.e., $z=0.023$ ) corresponding to the Coma Cluster (A1656). Since our grid resolution amounts to $\sim 200$ $h^{-1} \mathrm{kpc}$, at this distance the minimum size of a pixel of the synthetic image corresponds to $9^{\prime} .8$ square.

\section{RESULTS}

\subsection{Cosmic-Ray Energy Content}

The CR pressure at $\boldsymbol{x}_{i}$ is defined by

$$
P_{\mathrm{cr}}\left(\boldsymbol{x}_{i}\right)=\frac{4 \pi}{3} c \int_{p_{\mathrm{inj}}}^{p_{\max }} f\left(\boldsymbol{x}_{i}, p\right) \frac{p^{4}}{\left(m_{p}^{2} c^{2}+p^{2}\right)^{1 / 2}} d p
$$

with $f\left(\boldsymbol{x}_{i}, p\right)$ reconstructed from $n\left(\boldsymbol{x}_{i}, p\right)$ and $q\left(\boldsymbol{x}_{i}, p\right)$ as described in $\S 2.2$. From our simulations we find that $n\left(x_{i}, p\right)$ has a strong spatial dependence, whereas $q\left(\boldsymbol{x}_{i}, p\right)$ assumes a relatively narrow range of values, mostly between 4.01 and 4.2. The thermal pressure obeys by the equation of state for an ideal gas

$$
P_{\mathrm{th}}\left(\boldsymbol{x}_{i}\right)=n_{\text {gas }}\left(\boldsymbol{x}_{i}\right) k_{\mathrm{B}} T\left(\boldsymbol{x}_{i}\right),
$$

where $n_{\text {gas }}$ is the gas number density and $T$ the gas temperature. From the thermal and CR pressures defined at each cell, we calculate the mean thermal and CR pressure of groups/clusters within a sphere of radius $R_{\mathrm{cl}} \simeq 0.5 h^{-1} \mathrm{Mpc}$ from the cluster center as

$$
\left(\begin{array}{l}
P_{\mathrm{th}} \\
P_{\mathrm{cr}}
\end{array}\right)_{\mathrm{cl}}=\frac{1}{\sum_{i} w_{i}} \sum_{i} w_{i}\left[\begin{array}{l}
P_{\mathrm{th}}\left(\boldsymbol{x}_{i}\right) \\
P_{\mathrm{cr}}\left(\boldsymbol{x}_{i}\right)
\end{array}\right],
$$

where the summation over $i$ extends to the groups/clusters' volume $V=4 \pi R_{\mathrm{cl}}^{3} / 3$, and the weight function $w_{i}$ is given by the portion of each computational cell within $V$. Given our resolution, the volume within a radius of $0.5 h^{-1} \mathrm{Mpc}$ typically includes about 65 computational cells. We also 
calculate the groups/clusters' core temperature, $T_{x}$, as a volume-averaged ICM temperature within the same volume.

The first important result of this study is illustrated in Figure 1. There we plot the ratio $\left(P_{\mathrm{cr}} / P_{\mathrm{th}}\right)_{\mathrm{cl}}$ for groups/ clusters at the current epoch (i.e., $z=0$ ) as a function of the core temperature, $T_{x}$. The values of this ratio, $\left(P_{\mathrm{cr}} / P_{\mathrm{th}}\right)_{\mathrm{cl}} \simeq$ $\left(E_{\mathrm{cr}} / 2 E_{\mathrm{th}}\right)_{\mathrm{cl}}$, where $E$ stands for energy density, offers a firstorder indication of the relative importance of the two components for the dynamics of groups/clusters. From Figure 1, we can read that a significant fraction (up to $\sim 45 \%$ ) of the total pressure inside today's groups/clusters could be borne by CRs. We note here that the actual content of CR pressure (and energy density) depends on the injection parameter for which we have made an educated estimate on the basis of published studies related to the theory of diffusive shock acceleration. As already pointed out in $\S \S 1$ and 2.2, here we are allowed only a simplified treatment of the injection mechanism at shocks. Therefore, the result in Figure 1 should not be interpreted as a precise estimate of the CR content inside groups/clusters of galaxies. Rather, it provides a qualitative, yet sound, insight that CRs might be quite important for the dynamics of those objects. Considering the difficulties in following the physics of CR acceleration self-consistently in multidimensional simulations, the quantitative estimate of the total CR content needs to be done through measurements of $\gamma$-ray fluxes from groups/ clusters, as we shall see below.

The ratio of pressures plotted in Figure 1 does not show any particular trend except for a slight reduction toward higher temperatures. This might be due to our injection model, which, according to equation (2.5), produces a higher ratio of $\mathrm{CR}$ to thermal pressure for shocks with smaller velocities $\left(u_{s}\right)$ and similar Mach numbers [yielding similar $q(M)$ ]; i.e., cooler preshock gas. It is possible that such shocks occur in cooler groups/clusters, characterized by lower accretion velocities and similar preshock temperatures. However, the trend in Figure 1 could also be due

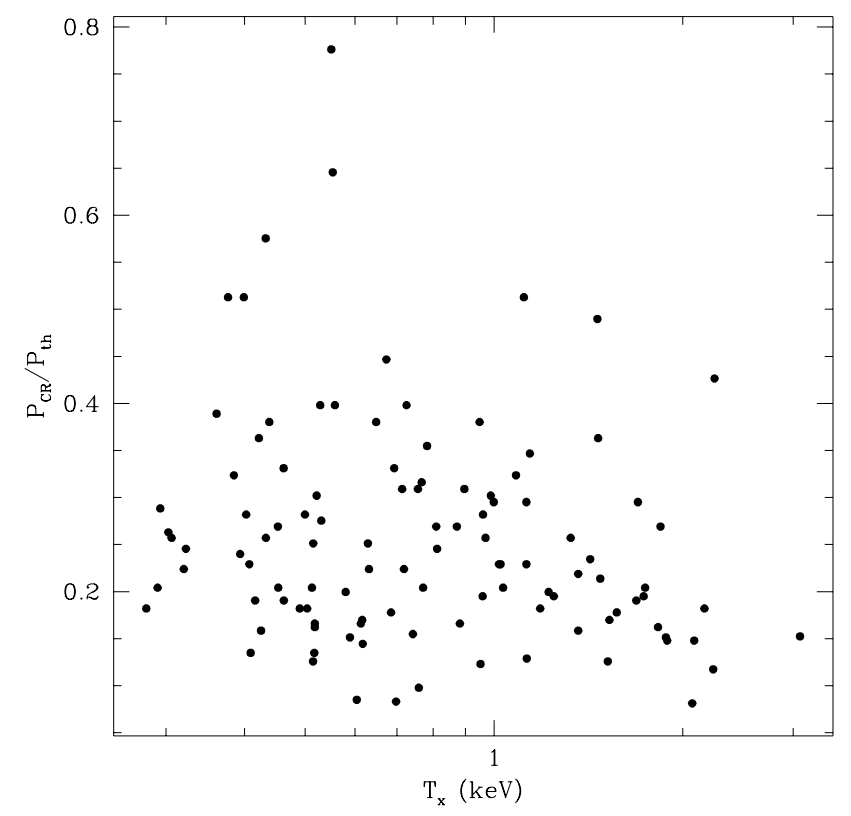

FIG. 1.- Ratio of CR to thermal pressure averaged over the group/ cluster volume within $0.5 h^{-1} \mathrm{Mpc}$ plotted as a function of group/cluster core temperature. to adiabatic compression inside the cluster, which increases the thermal pressure at a higher rate than the CR pressure. At least we can be sure that the apparent scatter there is in part a reflection of the diverse CR acceleration histories of groups/clusters. In addition, part of this scatter can also be due to the different spatial distribution of the thermal and CR components, as we shall see in $\S \S 3.2$ and 3.4.

\subsection{Spatial Distribution of Thermal and Cosmic-Ray Pressure}

Another feature of interest is the distributions of thermal and CR pressures inside groups/clusters. The difference in the distributions of the two pressure components is an important detail because, according to the condition for hydrostatic equilibrium

$$
\frac{d P_{\mathrm{tot}}(r)}{d r}=-\frac{G M_{\mathrm{cl}}(r) \rho_{\mathrm{gas}}(r)}{r^{2}}
$$

so it is the total pressure gradient that responds to the group/cluster mass enclosed in a volume of radius $r$. Thus, the spatial distribution (gradient) of $P_{\mathrm{cr}}$ is as important as the amount of CR energy content $\left(E_{\mathrm{cr}} \simeq 3 P_{\mathrm{cr}}\right)$ itself, once $P_{\text {cr }}$ becomes dynamically significant.

First, we consider the ratio of CR to thermal pressure evaluated within each computational cell inside groups/ clusters, i.e., $\left(P_{\mathrm{cr}} / P_{\mathrm{th}}\right)_{\text {cell }}$. The average of this quantity over the cells inside each group/cluster volume is plotted in the left panel of Figure 2, as a function of the group/cluster temperature. This is similar but not identical to $\left(P_{\mathrm{cr}} / P_{\mathrm{th}}\right)_{\mathrm{cl}}$ in Figure 1. The standard deviation of $\left(P_{\mathrm{cr}} / P_{\mathrm{th}}\right)_{\mathrm{cell}}$ values within each group/cluster is shown in similar fashion on the right panel of the same figure. It is clear that the dispersion around the average value is as large as the average itself, indicating a strong variation of $\left(P_{\mathrm{cr}} / P_{\mathrm{th}}\right)_{\mathrm{cell}}$ inside each group/cluster volume. Note that the gas temperature and the slope of the CR distribution, $q$, are approximately uniform within groups/clusters. So this pressure behavior should be a reflection of the different spatial distributions of gas and CRs in the simulation.

In order to quantify the difference in spatial distributions of the two pressures, we define a pressure-weighted mean square radius

$$
R_{I}^{2}=\frac{\sum_{i} P_{i} r_{i}^{2}}{\sum_{i} P_{i}}
$$

where $P_{i}$ and $r_{i}$ indicate the pressure (either thermal or CR) and the distance from the group/cluster center of the $i$ th computational cell, respectively. In Figure 3 we plot the ratio of $R_{I}$ relative to the $\mathrm{CR}$ and thermal pressure, i.e., $R_{I\left(p_{\text {cr }}\right)} / R_{I\left(p_{\text {th }}\right)}$. The plot shows that this ratio is close to 1 , with a marked tendency to values slightly larger than 1 . That is, CR pressure would be distributed more diffusely than gas pressure in groups/clusters. Caution is needed here since the diameter of the collapsed objects covers only about five computational cells. Thus, although the collapsed objects have been formed with adequate resolution to assure their basic properties, the fine details of their structures have not been captured. Nevertheless, the systematic difference could be connected to the mechanism of CR production. In fact, strong shocks in the simulations are more commonly located at the outskirts of the collapsed object. Thus, the ratio of CR to-thermal pressure is higher there, causing $R_{I\left(p_{\mathrm{cr})}\right.}$ to be slightly larger than $R_{I\left(p_{\mathrm{th}}\right)}$. 

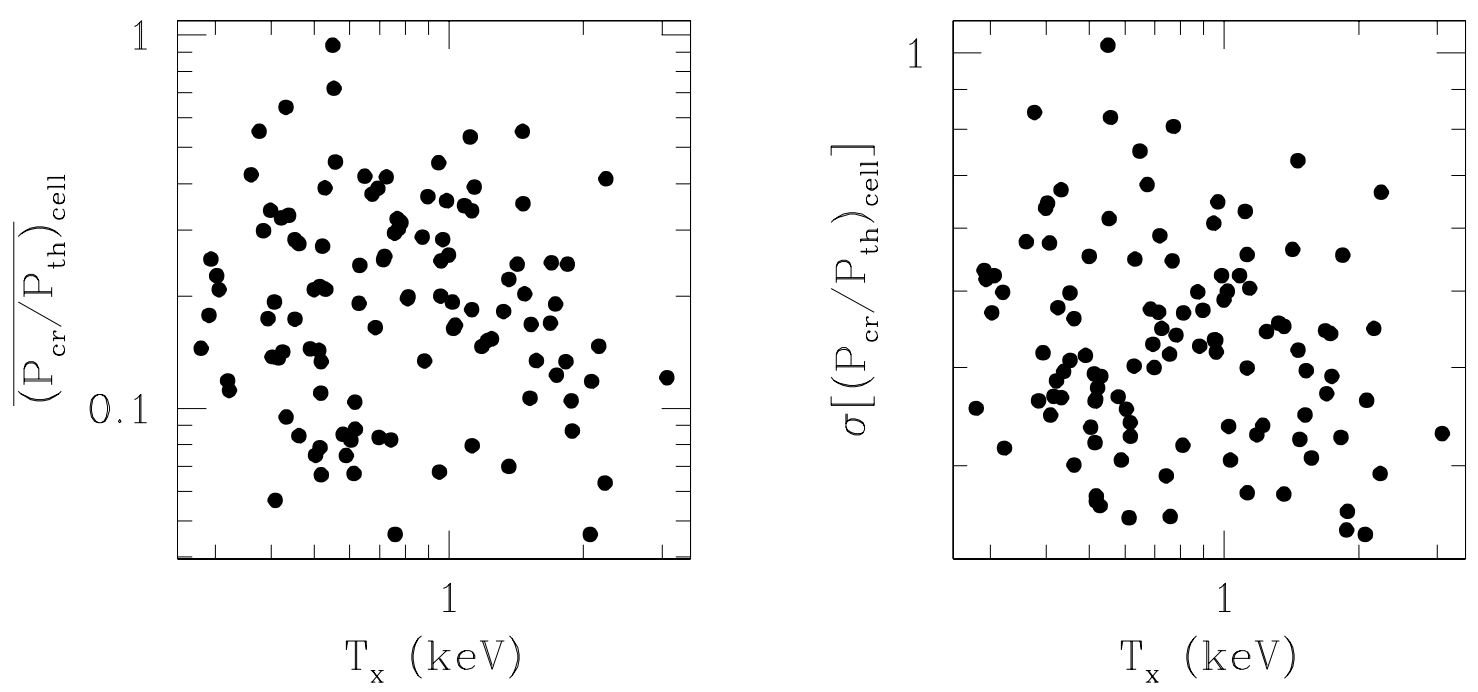

FIG. 2.-Left: Average of the cell-by-cell evaluation of $\left(P_{\mathrm{cr}} / P_{\mathrm{th}}\right)_{\mathrm{cell}}$. Right: Standard deviation.

\subsection{Gamma-Ray Flux}

A direct observational consequence of $\mathrm{CR}$ protons in groups/clusters is the $\gamma$-ray emission from $\pi^{0}$ decay. The $\gamma$-ray emissivity was calculated in each cell from the gas density and the CR proton distribution. The cross sections were computed according to the GALPROP routines (Moskalenko \& Strong 1998). The number of $\pi^{0}$ produced in each hadronic interaction, $\xi_{\pi^{0}}$, increases rather slowly with the proton energy, $E_{p}$, roughly as $\xi_{\pi^{0}} \simeq\left[\left(E_{p}\right.\right.$ $\left.\left.-E_{\mathrm{ths}}\right) / \mathrm{GeV}\right]^{1 / 4}$ for $E_{\mathrm{ths}} \lesssim E_{p} \lesssim 10^{4} \mathrm{GeV}\left(E_{\mathrm{ths}}=1.22 \mathrm{GeV}\right.$ is the energy threshold of the process; see, e.g., Mannheim \& Schlickeiser 1994). For a proton power-law distribution with kinetic energy, $f_{\mathrm{cr}}\left(T_{p}\right) \propto T_{p}^{-q+2}$, CRs at energy $T_{p}$ generate a number of $\pi^{0}$ that roughly scales as $j_{\pi^{0}} \propto$ $\left(T_{p} / \mathrm{GeV}\right)^{-(q-5 / 2)}$. Then, since $q \gtrsim 4$, the majority of the inte-

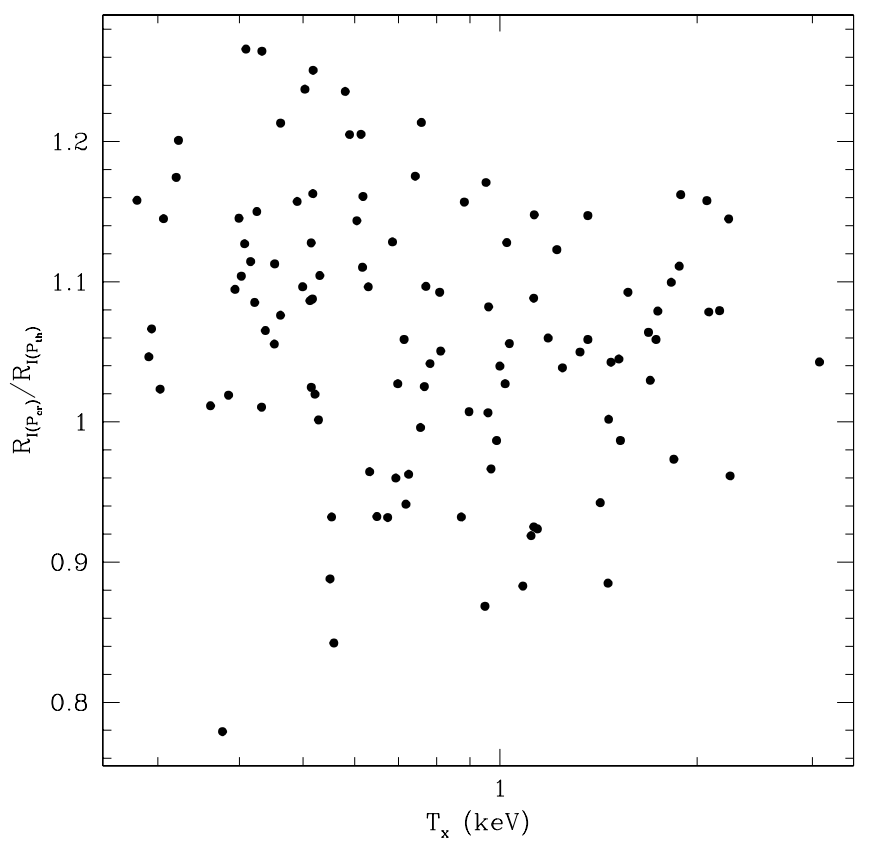

FIG. 3.-Ratio of pressure-weighted rms radii of CR to thermal pressure defined in eq. (3.5). grated $\gamma$-ray flux is contributed by CR protons in the lowenergy component.

In Figure 4 we report the expected $\gamma$-ray flux above 100 $\mathrm{MeV}, F_{\gamma}$, from a volume within $1.3 h^{-1} \mathrm{Mpc}$ from the group/cluster center, as a function of the core temperature, $T_{x}$. An integration volume larger than a typical group/ cluster core region of $0.5 h^{-1} \mathrm{Mpc}$ is chosen, because the CR proton distribution extends out farther to where the accretion shocks are found. When fitting the $F_{\gamma}-T_{x}$ relation with a power-law curve from a simple $\chi^{2}$ analysis, we get

$$
F_{\gamma}=7.4 \times 10^{-9}\left(\frac{T_{x}}{6.72 \mathrm{keV}}\right)^{2.95} \text { counts s}^{-1} \mathrm{~cm}^{-2} \text {. }
$$

We note here that the spread about the average at a given $T_{x}$ exhibited in Figure 4 is on the order of a few. This scatter,

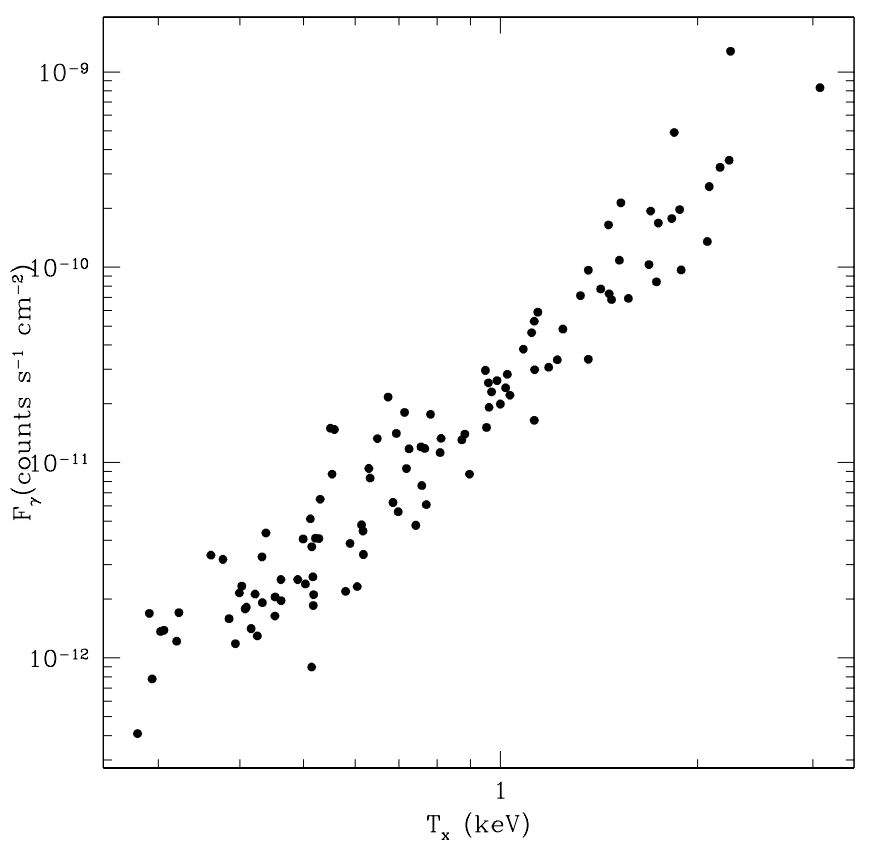

FIG. 4. $-\gamma$-ray flux as a function of group/cluster core temperature 
also pointed out in $\S 3.1$, is almost certainly real and is a reflection of the different peculiar formation history and current dynamical state that can characterize a group/ cluster with a given temperature. With this relation we find that for a Coma-like cluster with a temperature of $T_{x}=8.3$ $\mathrm{keV}$, the mean $\gamma$-ray flux would be $\sim 1.4 \times 10^{-8}$ counts $\mathrm{s}^{-1} \mathrm{~cm}^{-2}$. Similarly, after rescaling the flux for the appropriate distance, we can compute $F_{\gamma}$ expected for other clusters of known temperature. Thus, we find $F_{\gamma} \sim 9.8 \times 10^{-9}$ for a temperature of $6.3 \mathrm{keV}$ and a distance of $55 h^{-1} \mathrm{Mpc}$ characteristic of the Perseus Cluster (Schwarz et al. 1992), and we find $F_{\gamma} \sim 3.8 \times 10^{-9}$ for a temperature of $1.8 \mathrm{keV}$ and a distance of $14 h^{-1} \mathrm{Mpc}$ as estimated for the M87Virgo system (Böhringer et al. 1994).

The values found above for nearby clusters are well below the limit set by the EGRET experiment of $4 \times 10^{-8}$ counts $\mathrm{s}^{-1}$ (Sreekumar et al. 1996). However, they should be easily detectable by the Gamma-Ray Large-Area Space Telescope (GLAST), with a sensitivity an order of magnitude below the above value. Our estimate of the $\gamma$-ray fluxes is somewhat lower than the values computed by other authors (Dar \& Shaviv 1995; Ensslin et al. 1997), which are close to, or slightly in excess of, the EGRET upper limits. The estimated values are different, even after correcting our results for the aforementioned systematic underestimate owing to resolution effects. Differences in estimates of the $\gamma$-ray flux are expected, given the numerous differences in our physical assumptions and methodology when compared to the previous authors. For example, the spatial distribution of CRs given by the simulation in our case was assumed, by contrast, to be uniform in Dar \& Shaviv (1995), or such as to produce a constant ratio of $\mathrm{CR}$ to thermal pressure in Ensslin et al. (1997). In addition, we have used a fixed emitting volume within a radius of $1.3 h^{-1} \mathrm{Mpc}$, whereas the previous authors used $\sim 4 h^{-1} \mathrm{Mpc}$ for the size of the Coma Cluster (Dar \& Shaviv 1995; Ensslin et al. 1997) and 1.3 $h^{-1} \mathrm{Mpc}$ for a Virgo-like cluster (Dar \& Shaviv 1995). Also, for the slope of CR energy distribution, those authors (Dar \& Shaviv 1995; Ensslin et al. 1997) borrowed the empirical value from the Galactic case; i.e., they assumed $q=4.7$, unlike $q=4.0-4.2$ from our simulation.

Our estimate of the $\gamma$-ray flux for the Coma Cluster is, on the other hand, compatible with the value computed by Colafrancesco \& Blasi (1998), although our temperature dependence in equation (3.7) is quite a bit steeper than the one they presented. They computed the emission within the virial radius, whereas a fixed volume has been used in our estimate. In addition, they accounted analytically for a weak phenomenological dependence of the baryon fraction on the cluster size, while the baryon density from numerical simulation has been used in our estimate. We note that the expected functional form of the $\gamma$-ray flux can be modeled as

$$
F_{\gamma} \propto N_{\mathrm{cr}} n_{b},
$$

where $N_{\text {cr }}$ is the total number of CR protons and $n_{b}$ is the average group/cluster baryonic mass density, both inside a fixed radius. So most of the temperature scaling in our estimate is accounted for by the following facts:

1. The kinetic energy power available for CRs is proportional to $T_{x}^{2}$ (see Miniati et al. 2000). That is, $E_{\mathrm{cr}} \propto T_{x}^{2}$. On the other hand, with a constant momentum slope in the CR distribution function, $N_{\mathrm{cr}} \propto E_{\mathrm{cr}}$. Hence, $N_{\mathrm{cr}} \propto T_{x}^{2}$ holds approximately in the simulation.
2. The mean mass density inside a fixed volume (thus, also the associated baryon mass) scales almost linearly with the temperature, which is compatible with observations (e.g., Edge \& Steward 1991; Mohr, Mathiesen, \& Evrard 1999).

Together, these explain the origin of the $\sim T_{x}^{3}$ dependence found in the simulation presented here.

One important point in our findings is that the calculated values for the $\gamma$-ray flux are well below the upper limits set by EGRET, even while a large fraction of the total energy in the ICM gas is, in fact, stored in CRs. This result differs from Blasi (1999), who finds that the amount of energy in the CR component must be well below the equipartition value in order not to violate the same observational limits (except for the extreme case of a uniform distribution of CRs throughout clusters). Blasi's result derives from his adoption of a central point source for cluster CRs that then must diffuse throughout the cluster. That leads to a CR distribution more concentrated toward higher thermal gas densities in the core than if the CRs are produced by structure shocks, as in our case. Since in a denser environment the CRs experience many more interactions, a higher $\gamma$-ray flux is expected.

Finally, we have found that there is a tight correlation between the CR pressure and the $\gamma$-ray flux that can be fit by

$$
P_{\mathrm{cr}}=2.7 \times 10^{-11}\left(\frac{F_{\gamma}}{10^{-9} \text { counts s} \mathrm{cm}^{-2}}\right)^{0.64} \operatorname{ergs~cm}^{-3} .
$$

This is shown in Figure 5. The above scaling is compatible with $F_{\gamma} \propto T_{x}^{3}$ and $P_{\text {cr }} \propto T_{x}^{2}$, if the slope of the CR momentum distribution varies only slightly (as it is the case here); $P_{\text {cr }}-F_{\gamma}$ is manifestly a cleaner relation than $P_{\text {cr }}-T_{x}$ (not shown in this paper). The outcome is not surprising and is due to the direct physical relationship between $P_{\text {cr }}$ and $F_{\gamma}$,

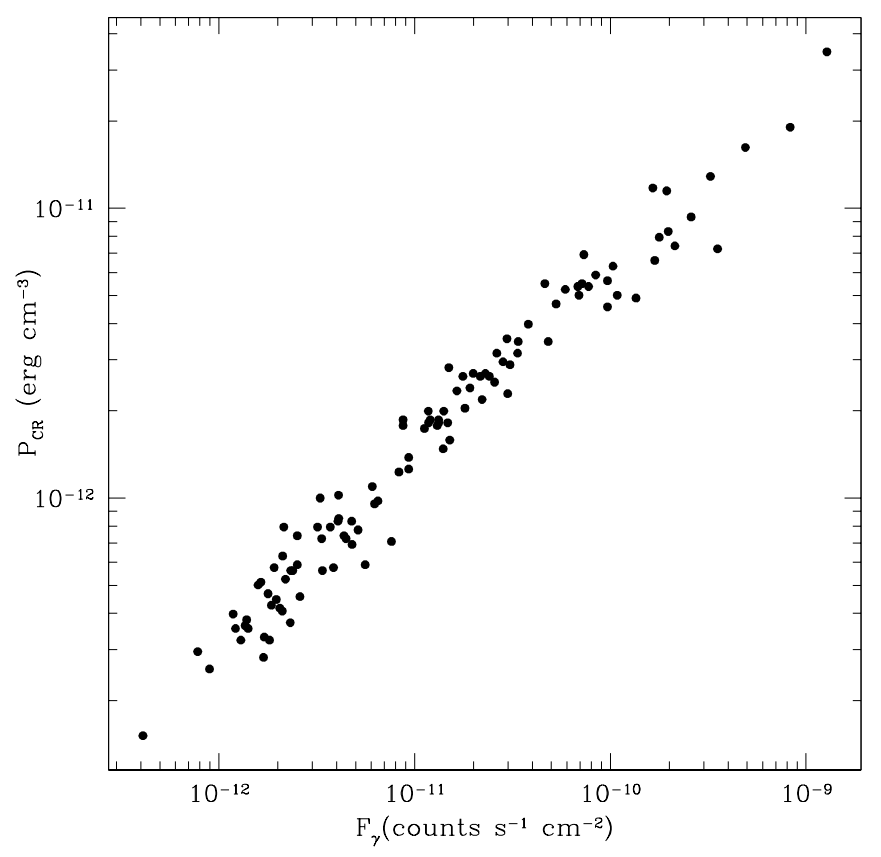

FIG. 5.-CR pressure as a function of $\gamma$-ray flux 
i.e., $F_{\gamma} \propto \int n_{\text {gas }} P_{\text {cr }}$ (for fixed $q$ ). Thus, the $P_{\mathrm{cr}}-F_{\gamma}$ relation probably allows the best determination of the amount of CR pressure in groups/clusters. In addition, measuring the $F_{\gamma}-T_{x}$ relation and comparing it to the numerical results will provide an important test for our numerical treatment and general understanding of CR injection, transport, and acceleration in group/cluster environment.

\section{4. $X$-Ray and Gamma-Ray Images}

In Figure 6, we present synthetic images of $\gamma$-ray emission from $\pi^{0}$ decay (left) and X-ray emission from thermal bremsstrahlung (right) for a rich group of galaxies in the simulation with $T_{x} \simeq 3 \mathrm{keV}$. A larger sample of groups/ clusters from the numerical simulation was studied by Miniati (2000), but their main properties can also be summarized by the findings below. The volume considered for the realization of the images in Figure 6 is a cube about 14 $h^{-1} \mathrm{Mpc}$ on a side, centered on the collapsed object. Each synthetic map has a gray-scale bar indicating the logarithmic value of the imaged quantity. The physical units are in ergs $\mathrm{cm}^{-2}$ pixel $^{-1}$ for the flux density of X-rays, and in counts $\mathrm{cm}^{-2}$ pixel $^{-1}$ for the flux density of $\gamma$-rays (see $\S 2.3$ for more details). In addition to the synthetic images, in Figure 7 we also present for the same object twodimensional slices of the following quantities: the CR proton number density in units of $\mathrm{cm}^{-3}$ (top left), the velocity field (top right), the contours of shock compression $(\nabla \cdot v$; bottom left $)$ and the gas number density in units of $\mathrm{cm}^{-3}$ (bottom right). The slices are through the object center and perpendicular to the line of sight of the synthetic images. The scale of the images in each panel of Figure 7 is about $5.3 h^{-1}$ Mpc.

First, we note that the synthetic $\gamma$-ray image exhibits an irregular morphology, somewhat different from the smooth slightly ellipsoidal shape of the thermal X-ray image. This is due to the fact that the CRs, responsible for the $\gamma$-ray emissivity, are sensitive to the particular shock distribution in the ICM. When even a mild shock crosses through a group/ cluster, the injection of fresh particles over a relatively short time can significantly enhance the CR population. This should definitely affect the shape of the $\gamma$-ray image. But it is not so for the X-ray image, because the effect of a weak shock is only a modest increase of the density (square) on which the emissivity primarily depends. That effect is likely to be blended away after line-of-sight integration (although finite resolution may limit the amount of visible features as well). In fact, closer inspection by means of two-dimensional slice images shows that enhanced CR density occurs downstream of "internal shocks," i.e., postshock flows in the central regions of groups/clusters (Miniati 2000). In Figure 7 the vertically elongated, high-CR density structure (top left) is enclosed by a Mach surface where a supersonic flow is suddenly decelerated by a shock. This region corresponds to a vertical dent in the velocity vector field (top right) in the northwest direction from the center of the panel. Note that the CRs are found where most of the intracluster gas is located (bottom right) in addition to being near the shock and, therefore, where the injection rate is higher. Such higher levels of structure and more irregular distribution of the CRs, as compared to the gas density, explain, in part, the high pixel-to-pixel fluctuation of the ratio of CR to thermal pressure that was found in $\S 3.2$.

Finally, we note that the regions of low surface brightness corresponding to the same factor below the peak value are slightly more extended in the $\gamma$-ray than in the X-ray image in accordance with findings in $\S 3.2$. Also, the highest $\gamma$-ray surface brightness appears more concentrated than the $\mathrm{X}$-ray brightness distribution. The X-ray emissivity is pro-
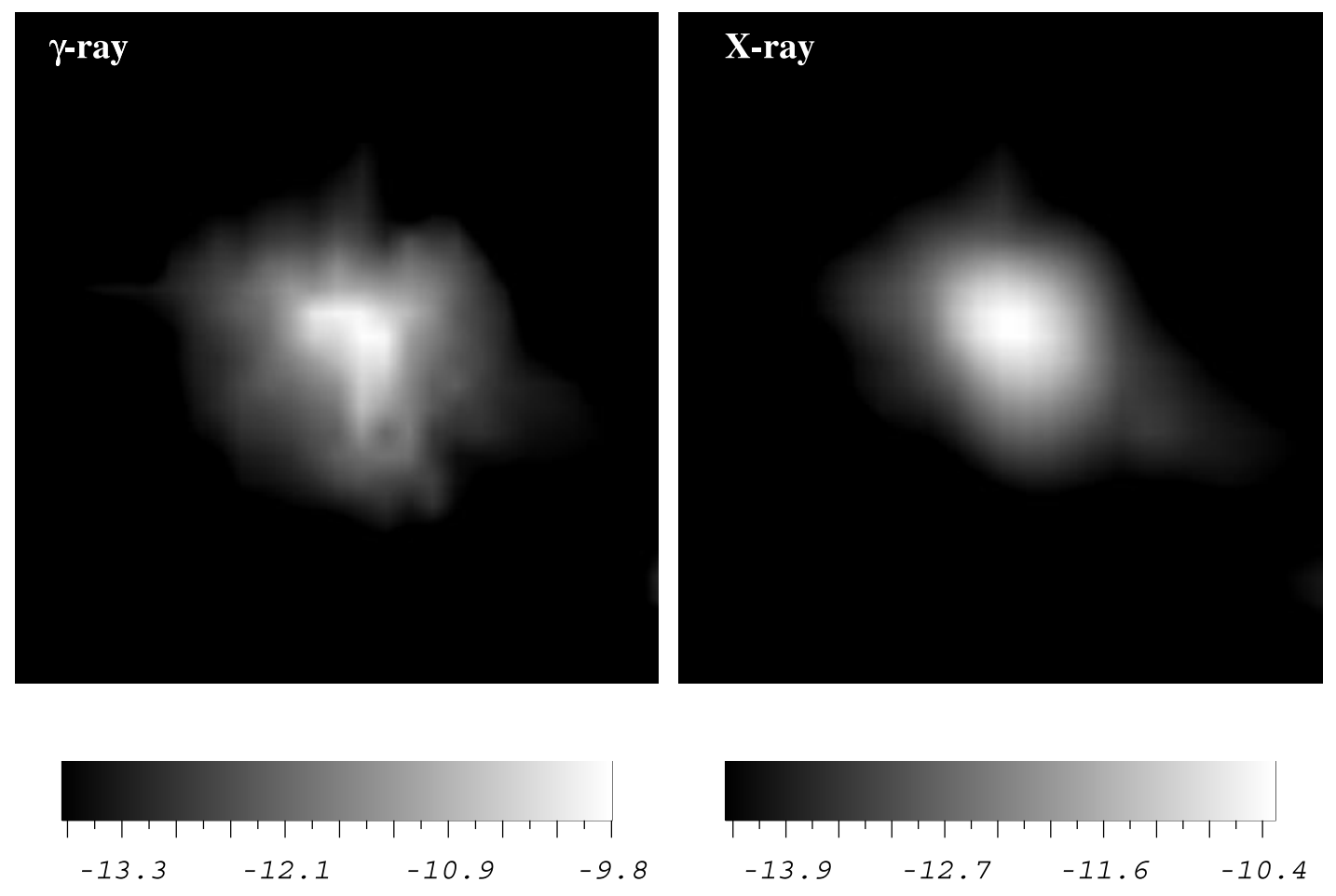

FIG. 6.-Synthetic images in $\gamma$-rays from $\pi^{0}$ decay in units of counts $\mathrm{s}^{-1} \mathrm{~cm}^{-2}$ pixel $^{-1}($ left $)$ and X-ray from thermal bremsstrahlung in units of ergs s $\mathrm{cm}^{-2}$ pixel $^{-1}$ (right) from a cosmic volume of $\left(14 h^{-1} \mathrm{Mpc}\right)^{3}$. 

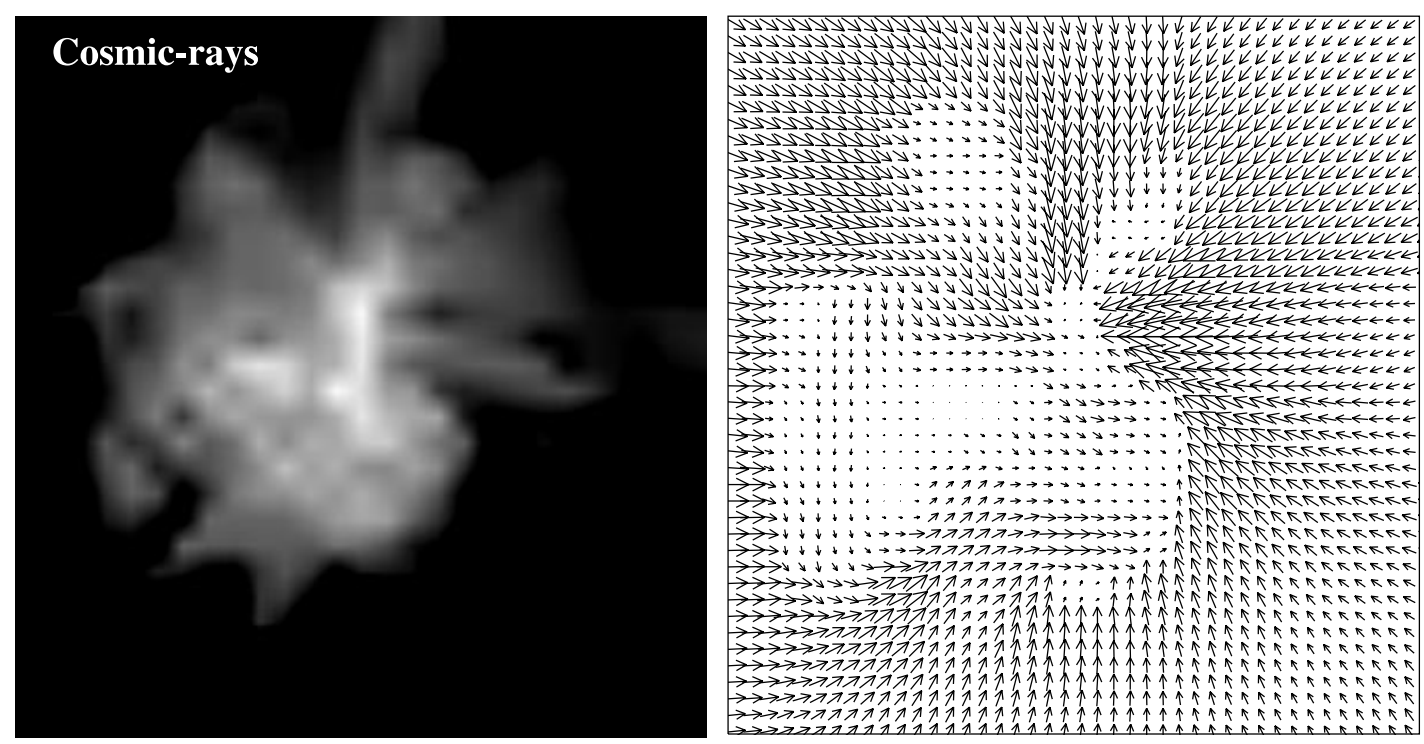

$\rightarrow=3.8391 e-02$

Velocity Field

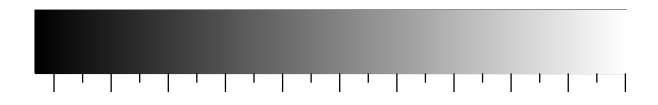

$\begin{array}{llll}-11.1 & -10.3 & -9.4 & -8.5\end{array}$
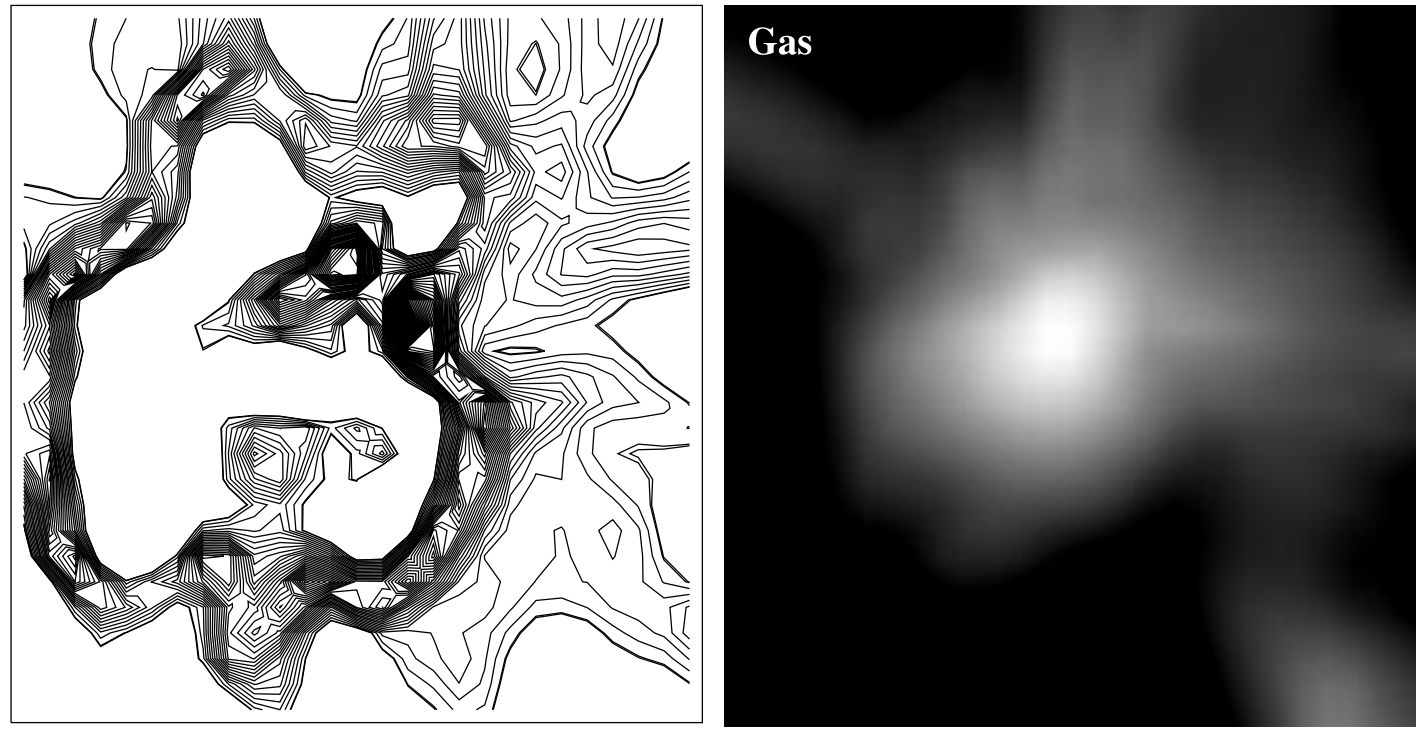

Shock Compression

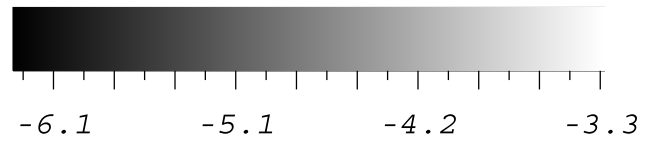

FIG. 7.-Two-dimensional slice maps of CR proton number density in units of $\mathrm{cm}^{-3}$ (top left), velocity field on that plane (top right), contours of shock compression $(\nabla \cdot v$; bottom left $)$ and gas number density, again in units $\mathrm{ofm}^{-3}$ (bottom right). The side of images is $5.3 h^{-1} \mathrm{Mpc}$.

portional to the square of the gas density, whereas the $\gamma$-ray emissivity is proportional to the product of the gas density with the CR density. Thus, this result simply means that the CR protons are slightly more concentrated by number than the gas in the group/cluster core region. This result does not contradict the finding in $\S 3.2$ that the CR pressure distribution is less concentrated than the gas pressure. Rather, it indicates that adiabatic compression is effective and has reduced the ratio $\left(P_{\mathrm{cr}} / P_{\mathrm{th}}\right)$ in the center of the collapsed objects.

\section{DISCUSSION}

As shown in the previous section, a significant fraction of the total energy associated with baryons inside a group/ cluster could be stored in CRs as a consequence of diffusive particle acceleration at structure formation shocks. This fact bears important consequences that we will try to address in the following discussion.

First, if the pressure provided by CRs, $P_{\text {cr }}=E_{\text {cr }} / 3$, is large enough, it can affect the dynamics of the ICM and, 
therefore, both its evolution and equilibrium. This is of great concern because groups/clusters of galaxies are invaluable probes to test cosmological theories and to measure key cosmological parameters (e.g., Bahcall et al. 1999 and references therein). In fact, the present-day abundance (number density) of rich clusters of galaxies sets a strong constraint on the total mass content of the universe and the normalization of the power spectrum of the density perturbation by imposing $\sigma_{8} \Omega_{m}^{1 / 2} \simeq 0.5 \pm 0.05$ (Bahcall \& Cen 1992; White, Efstathiou, \& Frenk 1993a; Eke, Cole, \& Frenk 1996; Viana \& Liddle 1996; Pen 1998). In addition, the evolution of the number density of rich clusters allows one to break the degeneracy of the above result and is used to determine both $\sigma_{8}$ and $\Omega_{m}$ (Carlberg et al. 1997; Bahcall, Fan, \& Cen 1997). It is not clear whether and how the evolution of structure would be affected by a nonthermal dynamical component. Clearly, since most of the mass is dark, the growth of the density perturbation would be unchanged for the most part. However, since the observable universe is made of baryons, the specific processes that determine their dynamical and thermal evolution are of crucial importance, as they also greatly affect cluster observational properties. In this respect, the effects produced by the CR component could be important, even though the underlying large-scale structure remains unaffected.

Furthermore, a substantial CR pressure component could contribute to the dynamical support of the ICM against gravitational collapse. This would affect the estimate of the total cluster mass derived from observations and, in turn, of both the baryonic fraction there and the total mass of the universe (White et al. 1993b). Results from a number of studies have suggested that mass estimates based on the hydrostatic equilibrium assumption and X-ray measurements tend to be somewhat smaller than those derived from virial estimates and gravitational lensing (Markevitch \& Vikhlinin 1997; Horner, Mushotzky, \& Scharf 1999; Nevalainen, Markevitch, \& Forman 2000; Roussel, Sadat, \& Blanchard 2000; Miralda-Escude \& Babul 1995; Wu 2000; see also Miniati 2000 for an extensive discussion on the issue). Part of this could well be the consequence of dynamical effects owing to the $\mathrm{CR}$ pressure and also the magnetic field pressure. However, the mass discrepancy issue is still controversial, and the precision of the current measurements, at the level of $20 \%$ accuracy, does not allow a strongly conclusive statement at this point. As discussed in the previous section, $\gamma$-ray observations appear promising in this respect, since according to our prediction the expected $\gamma$-ray flux from Coma-like clusters should be well above the detection threshold of GLAST (see also Blasi 1999; Dolag \& Ensslin 2000).

From the theoretical side, recent numerical simulations have shown that in order to construct a viable and realistic depiction of the ICM, the various processes taking place there need to be accounted for in sufficient detail. In particular, it has become clear that the effect of radiative cooling in cluster cores produces significant quantitative differences in numerical simulations in which it is allowed (e.g., Katz \& White 1993; Suginohara \& Ostriker 1998; Pearce et al. 2000; Lewis et al. 2000). In particular, Suginohara \& Ostriker (1998) found that cooling can become catastrophic in the cluster cores unless prevented by some additional physical processes. Similarly, Lewis et al. (2000) found that radiative cooling can have dynamical effects on the cluster structure and evolution. In particular, they concluded that the consequences of cooling are global and affect the cluster as a whole, despite the fact that strong cooling is localized in the central region of a cluster. According to the study by Lewis et al. (2000), however, the catastrophic character emerging in Sughinohara \& Ostriker's simulations is largely inhibited by the feedback of star formation (gas removal and heating). Nevertheless, that does not solve the cooling problem completely. In fact, the star formation ensuing from the cooling of the gas produces too large a stellar component $(30 \%$ of all the baryons instead of the observed $10 \%$ fraction), too high an X-ray luminosity by a factor of $\sim 3$, and too high a velocity dispersion (Lewis et al. 2000). These excesses are driven by a very high density, stellardominated core resulting from the effect of cooling. In this respect, the presence of a significant CR component could reduce the overly dramatic effect of radiative cooling and recover some of the observed cluster properties in at least two ways. First, CRs provide an additional nonthermal pressure that is not dissipated by radiative effects. This hinders the contraction of the cooling gas, therefore prolonging the cooling time and decreasing the rate of conversion of gas into stars. Second, low-energy CR ions provide a source of heating that tends to balance cooling, once again softening the effect of the latter (Rephaeli 1977). In this respect, CRs are also likely to affect the dynamics of a cooling flow by means of the two generic mechanisms described above.

\section{CONCLUSIONS}

We have carried out a computational study of production of CR protons at cosmological shocks associated with the large-scale structure in an SCDM universe. We have achieved this by carrying out the first numerical simulation of structure formation that directly includes shock acceleration (in the test-particle limit approximation), transport, and energy losses of the CRs. CR injection takes place at shocks according to the thermal leakage prescription, leading to the injection as CR of a fraction of about $10^{-4}$ of the thermal protons passing through a shock. According to our results, CR ions may provide a significant fraction of the total pressure in the ICM. The conclusion cannot be made strictly quantitative yet because the complex physics regulating the acceleration mechanism cannot be fully simulated, and our simulated group/cluster structures are still rather coarse. However, we expect the CR pressure may account for a few tens of percent of the total ICM pressure. The cosmological consequences of this result were addressed in $\S 4$.

A major step forward will be made possible by the advent of the next generation of $\gamma$-ray facilities, i.e., GLAST. In fact, we expect $\gamma$-rays will be detected for relatively nearby massive clusters. That development will directly probe the CR content in clusters of galaxies (see $\S 3.3$ ). In addition, $\gamma$-ray imaging and spectroscopy will enable us to infer the spatial distribution of the CR density and pressure once the gas distribution is known (e.g., through X-ray data). That will translate into direct information on the nature of the CR sources. In fact, if most of the CRs have been expelled by active galaxies, then their distribution would not be as widespread as in the case where the primary sources are cosmic shocks. This adds to the wealth of critical information provided by observation in this band. However, most probably only the largest clusters and only their innermost regions of highest emission will be probed by these 
instruments, owing to the very low surface brightness in the $\gamma$-ray band. Nevertheless, those detections would still be invaluable for the study and a much deeper understanding of the dynamics of these objects within the next few years.

F. M. wishes to acknowledge a Doctoral Dissertation Fellowship from the University of Minnesota. The work of F. M. and T. W. J. has been supported in part by NASA grant NAG 5-5055, by NSF grants AST 96-16964 and AST 00-71167, and by the University of Minnesota Supercomputing Institute. D. R. and H. K. were supported in part by grant 1999-2-113-001-5 from the interdisciplinary Research Program of the KOSEF. We thank T. Ensslin and S. D. M. White for useful comments on the manuscript. We are grateful to I. L. Tregillis for his ray-tracing code and to I. V. Moskalenko and A. W. Strong for providing their GALPROP routines.

\section{REFERENCES}

Bahcall, N. A. 1999, in Formation of Structure in the Universe, ed. A. Dekel \& J. P. Ostriker (Cambridge: Cambridge University Press), 135 Bahcall, N. A., \& Cen, R. 1992, ApJ, 398, L81

Bahcall, N. A., Fan, X., \& Cen, R. 1997, ApJ, 485, L53

Bahcall, N. A., Ostriker, J. P., Perlmutter, S., \& Steinhardt, P. J. 1999, Science, 284, 1481

Berezhko, E. G., \& Ellison, D. C. 1999, ApJ, 526, 385

Berezhko, E. G., Ksenofontov, L. T., \& Yelshin, V. K. 1995, Nucl. Phys. B, 39,171

Berezinsky, V. S., Blasi, P., \& Ptuskin, V. S. 1997, ApJ, 487, 529

Bertschinger, E. 1985, ApJS, 58, 39

Blasi, P. 1999, ApJ, 525, 603

Böhringer, H., Briel, U. G., Schwarz, R. A., Voges, W., Hartner, G., \& Trumper, J. 1994, Nature, 368, 828

Carlberg, R. G., Morris, S. L., Yee, H. K. C., \& Ellingson, E. 1997, ApJ, 479, L19

Cen, R., \& Ostriker, J. P. 1999, ApJ, 517, 31

Colafrancesco, S., \& Blasi, P. 1998, Astropart. Phys., 9, 227

Dar, A., \& Shaviv, N. J. 1995, Phys. Rev. Lett., 75, 3052

Dolag, K., \& Ensslin, T. 2000, A\&A, 362, 151

Drury, L. O. 1983, Rep. Prog. Phys., 46, 973

Edge, A. C., \& Steward, G. C. 1991, MNRAS, 252, 414

Eichler, D. 1979, ApJ, 229, 419

Eke, V. R., Cole, S., \& Frenk, C. S. 1996, MNRAS, 282, 263

Ellison, D. C., \& Eichler, D. 1984, ApJ, 286, 691

Ensslin, T. A., Biermann, P. L., Klein, U., \& Kohle, S. 1998, A\&A, 332, 395

Ensslin, T. A., Biermann, P. L., Kronberg, P. P., \& Wu, X.-P. 1997, ApJ, 477,560

Frenk, C. S., et al. 1999, ApJ, 525, 554

Gieseler, U. D. J., Jones, T. W., \& Kang, H. 2000, A\&A, 364, 911

Horner, D. J., Mushotzky, R. F., \& Scharf, C. A. 1999, ApJ, 520, 78

Jones, T. W., Miniati, F., Ryu, D., \& Kang, H. 2000, in AIP Conf. Proc. 558, High-Energy Gamma-Ray Astronomy, ed. F. Aharonian \& H. Völk, (New York: AIP), 436

Jones, T. W., Ryu, D., \& Engel, A. 1999, ApJ, 512, 105

Kang, H., \& Jones, T. W. 1995, ApJ, 447, 944

Kang, H., Ostriker, J. P., Cen, R., Ryu, D., Hernquist, L., Evrard, A. E., Bryan, G. L., \& Norman, M. L. 1994, ApJ, 430, 83

Kang, H., Rachen, J., \& Biermann, P. L. 1997, MNRAS, 286, 257

Kang, H., Ryu, D., \& Jones, T. W. 1996, ApJ, 456, 422

Katz, N., \& White, S. D. M. 1993, ApJ, 412, 455

Kronberg, P. P. 1994, Rep. Prog. Phys, 57, 325

Kulsrud, R. M., Cen, R., Ostriker, J. P., \& Ryu, D. 1997, ApJ, 480, 481

Lacey, C., \& Cole, S. 1994, MNRAS, 271, 676
Lee, M. A. 1982, J. Geophys. Res., 87, 5063

Lewis, G. F., Babul, A., Katz, N., Quinn, T., Hernquist, L., \& Weinberg, D. H. 2000 , ApJ, 536, 623

Malkov, M. A., \& Völk, H. J. 1995, A\&A, 300, 605

Mannheim, K., \& Schlickeiser, R. 1994, A\&A, 286, 983

Markevitch, M., Sarazin, C. L., \& Vikhlinin, A. 1999, ApJ, 521, 526

Markevitch, M., \& Vikhlinin, A. 1997, ApJ, 491, 467

Miniati, F. 2000, Ph.D. thesis, Univ. Minnesota

2001, Comp. Phys. Comm., in press

Miniati, F., Jones, T. W., Kang, H., \& Ryu, D. 2001, ApJ, in press

Miniati, F., Ryu, D., Kang, H., Jones, T. W., Cen, R., \& Ostriker, J. 2000, ApJ, 542, 608

Miralda-Escude, J., \& Babul, A. 1995, ApJ, 449, 18

Mohr, J. J., Mathiesen, B., \& Evrard, A. E. 1999, ApJ, 517, 627

Moskalenko, I. V., \& Strong, A. W. 1998, ApJ, 493, 694

Nevalainen, J., Markevitch, M., \& Forman, W. 2000, ApJ, 532, 694

Ostriker, J. P. 1993, ARA\&A, 31, 689

Ostriker, J. P., \& Cen, R. 1996, ApJ, 464, 27

Pearce, F. R., Couchman, H. M. P., Thomas, P. A., \& Edge, A. C. 2000, MNRAS, 317, 1029

Pen, U.-L. 1998, ApJ, 498, 60

Quest, K. B. 1988, J. Geophys. Res., 93, 9649

Quilis, V., Ibanez, J. M. A., \& Saez, D. 1998, ApJ, 502, 518

Rephaeli, Y. 1977, ApJ, 212, 608

Roettiger, K., Burns, J. O., \& Stone, J. M. 1999, ApJ, 518, 603

Roussel, H., Sadat, R., \& Blanchard, A. 2000, A\&A, 361, 429

Ryu, D., \& Kang, H. 1997, MNRAS, 284, 416

Ryu, D., Kang, H., \& Biermann, P. L. 1998, A\&A, 335, 19

Ryu, D., Ostriker, J. P., Kang, H., \& Cen, R. 1993, ApJ, 414, 1

Sarazin, C. L. 1999, ApJ, 520, 529

Schwarz, R. A., Edge, A. C., Voges, W., Böhringer, H., Ebeling, H., \& Briel, U. G. 1992, A\&A, 256, L11

Sreekumar, P., et al. 1996, ApJ, 464, 628

Suginohara, T., \& Ostriker, J. P. 1998, ApJ, 507, 16

Sunyaev, R. A., \& Zeldovich, Y. B. 1972, A\&A, 20, 189

Takizawa, M., \& Naito, T. 2000, ApJ, 535, 586

Viana, P. T. P., \& Liddle, A. R. 1996, MNRAS, 281, 323

Völk, H. J., Atoyan, A. M., \& Breitschwerdt, D. 1996, Space Sci. Rev., 75, 279

White, S. D. M., Efstathiou, G., \& Frenk, C. S. 1993a, MNRAS, 262, 1023

White, S. D. M., Navarro, J. F., Evrard, A. E., \& Frenk, C. S. 1993b, Nature, 366,429

Wu, X.-P. 2000, MNRAS, 316, 299 